Revisión y/o Actualización de Literatura

\title{
El abordaje de terapia ocupacional considerando los activos para la salud en población joven: un análisis de la literatura
}

\author{
A abordagem da terapia ocupacional considerando os ativos para a saúde \\ da população jovem: uma análise da literatura
}

\section{Occupational therapy considering health assets in young population: a scoping review}

\author{
Rut Alsina-Santana ${ }^{a}$ (D), Inmaculada Zango-Martín ${ }^{b}$ (D) \\ ${ }^{a}$ Instituto Guttmann de Barcelona, Barcelona, España. \\ bescuela Universitaria de Enfermería y Terapia Ocupacional (EUIT), Universidad Autónoma de Barcelona. Espańa.
}

Cómo citar: Alsina-Santana, R., \& Zango-Martín, I. (2022). El abordaje de terapia ocupacional considerando los activos para la salud en población joven: un análisis de la literatura. Cadernos Brasileiros de Terapia Ocupacional, 30, e2967. https://doi.org/10.1590/2526-8910.ctoAR22482967

\begin{abstract}
$\underline{\text { Resumen }}$
Introducción: La salutogénesis es una orientación académica que busca resaltar los factores que promueven activamente la salud y el bienestar de las personas, ofreciendo un mayor control sobre sus vidas y su salud. Dentro de este enfoque destacan los activos para la salud de la población disponibles en la comunidad. Objetivo: Revisar la literatura sobre las principales ocupaciones o actividades significativas considerando el planteamiento de los activos para la salud en jóvenes. Método: Se realizó una revisión de la literatura en las bases de datos PubMed, Science Direct (Elsevier) y Biblioteca Virtual en Salud, entre los ańos 2013-2019. Se utilizaron los descriptores "health assets" AND (Children OR Adolescents) y "Activos para la salud" AND jóvenes. Se escogieron 10 artículos de los 145 cribados. Resultados: Los diez artículos fueron seleccionados y analizados. Los activos para la salud más relevantes en los jóvenes son: el autoconocimiento y la autoconfianza. En cuanto a la ocupación, consideran que llevar un estilo de vida saludable, tener una vida con propósito, el ocio y las relaciones sociales son los principales activos para la salud. Por otra parte, se ha identificado un fuerte impacto de la relación con la familia para los activos para la salud. Conclusión: En la intervención de terapia ocupacional es fundamental incluir a la población joven con la identificación de los activos para la salud y cómo impactan en la salud y el bienestar. Además, es importante investigar de qué modo los activos para la salud pueden reducir la desigualdad social de la comunidad. Por otra parte, se ha observado que los activos para la salud descritos por los jóvenes pueden presentar influencias por parte de la sociedad que, en vez de aportar salud, pueden ser nocivas para ellos.
\end{abstract}


Palabras-clave: Jóvenes, Promoción de la Salud, Participación de la Comunidad, Terapia Ocupacional, Actividades Cotidianas.

\section{$\underline{\text { Resumo }}$}

Introduçáo: A salutogênese é uma orientação acadêmica que busca ressaltar os fatores que promovem ativamente a saúde e o bem-estar das pessoas, proporcionando maior controle sobre sua vida e saúde. Nesse enfoque se destacam os ativos para a saúde da populaçáo disponíveis na comunidade. Objetivo: Apresentar uma revisão da literatura sobre as principais ocupaçóes ou atividades significativas considerando a abordagem dos ativos para a saúde em jovens. Método: Foi realizada uma revisão da literatura nas bases de dados PubMed, Science Direct (Elsevier) e Biblioteca Virtual em Saúde, entre os anos de 2013-2019. Foram utilizados os descritores "health assets" AND (Children OR Adolescents) e "Activos para la salud" AND jóvenes. Dez artigos foram escolhidos entre os 145 selecionados. Resultados: Dez artigos foram selecionados e analisados. Os ativos de saúde mais relevantes identificados nos jovens foram: autoconhecimento e autoconfiança. Em relação à ocupaçấo, consideram que levar um estilo de vida saudável, ter uma vida com propósito, lazer e relaçôes sociais são os principais ativos de saúde identificados. Por outro lado, identificou-se forte impacto da relaçáo com a família para bens de saúde. Conclusão: É fundamental incluir na intervenção da terapia ocupacional com a populaçáo jovem a identificaçáo dos ativos de saúde e como eles impactam a saúde e o bem-estar. Além disso, é importante investigar como os ativos de saúde podem reduzir a desigualdade social da comunidade.

Palavras-chave: Juventude, Promoção da Saúde, Participação da Comunidade, Terapia Ocupacional, Atividades Cotidianas.

\section{$\underline{\text { Abstract }}$}

Introduction: Salutogenesis is an academic orientation that seeks to highlight the factors that actively promote the health and well-being of people, offering greater control over their lives and health. Within this approach, the assets for the health of the population available in the community stand out. Objective: Review the literature on the main occupations or significant activities considering the approach of assets for health in young people. Method: A literature review was carried out in the PubMed, Science Direct (Elsevier) and Virtual Health Library databases, between the years 2013-2019. The descriptors "health assets" AND (Children OR Adolescents) and "Activos para la salud" AND jóvenes were applied. Ten articles were chosen from the 145 screened. Results: Ten articles were selected and analysed. The most relevant health assets identified in young people are self-knowledge and self-confidence. Regarding occupation, they consider that leading a healthy lifestyle, having a purposeful life, leisure and social relationships are the main identified health assets. On the other hand, a strong impact of the relationship with the family for health assets has been identified. Conclusion: It is essential to include in the occupational therapy intervention with the young population the identification of health assets and how they impact health and well-being. In addition, it is important to investigate how health assets can reduce community social inequality.

Keywords: Youth, Health Promotion, Community Participation, Occupational Therapy, Activities of Daily Living. 


\section{Introducción}

La salutogénesis es un enfoque de salud surgido en 1979 cuya finalidad es analizar los orígenes de la salud y los activos para la salud que previenen la enfermedad y promueven la salud y el bienestar (Hernán et al., 2013; Mittelmark et al., 2017). Según Mittelmark et al. (2017), esta orientación se basa en las fortalezas de las personas y sus entornos, dejando de lado los déficits. Así pues, la salutogénesis busca resaltar los factores que promueven activamente la salud y el bienestar, ofreciendo a las personas un mayor control sobre sus vidas y su salud (Hernán et al., 2013). Según Antonovsky, todos estamos expuestos a factores estresantes que nos provocan tensión. No obstante, son los recursos generales de resistencia de cada persona (psicológicos, sociales y culturales) los que facilitan el manejo de las situaciones vitales evitando la enfermedad (Hernán et al., 2013; Mittelmark et al., 2017). A partir de 2017 este planteamiento de los activos para la salud comienza a cobrar relevancia gracias a las jornadas sobre activos en salud comunitaria gestionadas por la Escuela Andaluza de Salud Pública de Granada (Mittelmark et al., 2017; Sociedad Española de Salud Pública y Administración Sanitaria, 2017). Con la finalidad de identificar los principales activos para la salud entre la población joven centrándonos especialmente en las actividades significativas y su vínculo con la salud y el bienestar, planteamos abordar en primer lugar aspectos generales sobre los activos para la salud en población joven y, en segundo lugar, el planteamiento de los activos para la salud desde una perspectiva ocupacional.

\section{Relevancia del abordaje de activos para la salud en población joven}

Los activos para la salud se entienden como cualquier factor o recurso disponibles en la comunidad, que actúa como un factor de protección y promoción, para amortiguar las tensiones y el estrés diario, mantener y sostener la salud y el bienestar y reducir las desigualdades en salud entre los ciudadanos, grupos y comunidades (Cofiño et al., 2016; Royal College of Occupational Therapists, 2017; Hernán et al., 2013). Según el Observatorio de Salud de Asturias (2017) para que un recurso de la comunidad sea un activo para la salud, éste ha de ser conocido, valorado positivamente y utilizado por las personas de una comunidad.

Además de los activos para la salud, otro de los conceptos clave del modelo salutogénico, es el Sentido de Coherencia (SOC, por sus siglas en inglés). Este se puede definir como la experiencia vital de aportar recursos para hacer frente a los factores estresantes, que genera un sentimiento de confianza en entornos predecibles y comprensibles que ayuda a hacer frente a los desafíos derivados de los estímulos internos y externos. El SOC ayuda a la persona a movilizar Recursos de Resistencia (RR) frente a los estresores psicosociales y físicos (Hernán et al., 2013; Mittelmark et al., 2017). Así, los RR están formados por cualquier característica de la persona, la comunidad o el entorno que facilite el manejo de la tensión y ayuden a percibir la vida como coherente, estructurada y comprensible. Estos, deben estar a disposición de la población y pueden ser psicológicos, socioculturales y/o del entorno físico y natural (Hernán et al., 2013; Mittelmark et al., 2017). Teniendo esto en cuenta, es importante considerar que todas las personas estamos expuestos a factores estresantes que nos generan tensión, si no contamos con recursos de resistencia esta tensión se convierte en estrés y genera enfermedades. 
Por el contrario, si entendemos lo que nos está ocurriendo podemos identificar los recursos necesarios para afrontarlo y valorar si tenemos o no la capacidad de usarlos para modificar los efectos de los factores estresantes; esto tiene un impacto determinante en el afrontamiento y manejo de la tensión para evitar la enfermedad (Hernán et al., 2013). De este modo, considerar los recursos de resistencia (RR) fortalecen el SOC de la persona generando más oportunidades para hacer frente a los retos de la vida. El SOC está compuesto por tres componentes clave: comprensión, manejabilidad y significatividad (Hernán et al., 2013; Mittelmark et al., 2017).

El principal descubrimiento del modelo salutogénico es la correlación entre el SOC con la salud y la calidad de vida percibidas (Hernán et al., 2013). Según Rodríguez \& García (2005 en Souto Gómez et al., 2019), la calidad de vida

[...] es una necesidad definida en satisfacer las actividades de la vida diaria básicas o instrumentales, el trabajo, el ocio o la participación social, entre otras, que depende de las percepciones y valoración personal, así como del ambiente donde las personas, poblaciones o comunidades desempeñen su ocupación (Souto Gómez et al., 2019, p. 52).

Así pues, la calidad de vida será percibida como positiva o negativa dependiendo de los valores que presente la persona y las demandas generadas por su entorno. Los beneficios para la salud que se derivan de este enfoque son (Observatorio de Salud de Asturias, 2017, p. 8): reducción del aislamiento y soledad, aumento de la autoestima y confianza, sentido de control y empoderamiento, aumento del bienestar psicológico o mental, disminución de síntomas de ansiedad y/o depresión y del estado de ánimo negativo, aumento en la sociabilidad, habilidades de comunicación y conexiones sociales, mejora en la motivación y el significado de la vida dando esperanza y optimismo sobre el futuro, mejora en la salud física y un estilo de vida más saludable y la incorporación de aprendizajes, intereses y habilidades, incluyendo habilidades artísticas.

Para llevar a cabo este enfoque, previamente, se genera un mapa de activos para la salud. En este mapa se identifican los recursos positivos del entorno para crear un inventario de las fortalezas y capacidades de los ciudadanos de esa comunidad, sustentado en las potencialidades y los intereses de la comunidad. Este inventario se centra en los recursos que tienen las personas para generar salud y resalta las conexiones entre ellos como forma de empoderarse y renovarse en un entorno concreto. Tal y como determinan Hernán, Morgan \& Mena (2013, p. 54), “[...] cuanto mejor conoce la persona el mundo en el que vive [...] mejor podrá utilizar los recursos que posee y que hay a su alrededor para conservar su propia salud" (Cofiño et al., 2016; Hernán et al., 2013). Una vez identificados los activos para la salud de una comunidad, los servicios de Atención Primaria realizan una recomendación de activos o "prescripción social". Esta prescripción implica recomendar recursos de la comunidad que están vinculados con el bienestar y la salud de la persona (Observatorio de Salud de Asturias, 2017).

\section{Los activos para la salud desde una perspectiva ocupacional}

En el marco de la terapia ocupacional, el enfoque basado en activos para la salud, derivado de la teoría salutogénica, tiene el objetivo de "[...] promover y fortalecer los factores que apoyan la buena salud y el bienestar, permitiendo a esas personas, grupos y 
comunidades obtener más control sobre sus vidas y circunstancias" (Royal College of Occupational Therapists, 2017, p. 5). De este modo, la terapia ocupacional contribuye a dar sentido a la vida de las personas y favorece el bienestar a partir de promover la participación de las personas en su comunidad y en las actividades de su vida cotidiana, implicándoles en la prevención, la promoción y la restauración de su identidad ocupacional (Souto Gómez et al., 2019).

En este sentido, se entiende el bienestar, según Souto Gómez et al. (2019), como la percepción personal de encontrarse en un estado óptimo de salud, a partir del cual puedes desarrollarte como individuo respondiendo a las expectativas de los roles demandados por el entorno en el que vives. Por lo tanto, el bienestar está estrechamente vinculado con la identidad ocupacional de las personas (Algado, 2015; Souto Gómez et al., 2019; Zango Martín, 2011). Así, siguiendo este enfoque de salud, en el ámbito de la terapia ocupacional se plantea la justicia ocupacional como el derecho de todas las personas de disfrutar de oportunidades para desarrollar sus ocupaciones, en la que cuenten con los recursos necesarios para alcanzar su potencial y experimentar bienestar (Parra-Esquivel, 2015 en Souto Gómez et al., 2019). Cuando esto no ocurre, se genera una situación de desequilibrio, marginación, alineación y/o privación ocupacional (Souto Gómez et al., 2019; Zango Martín, 2017). Para considerar esta situación es clave tener en cuenta el contexto en el que se desarrollan las ocupaciones y de qué modo estos promueven o limitan la participación y por lo tanto la salud y el bienestar (Zango Martín, 2017).

El modelo salutogénico del que habla Antonovsky se centra en la importancia de los contextos para la salud (Cantero Garlito, 2018). Según Cantero Garlito (2018), desde los inicios de la terapia ocupacional, se le ha otorgado relevancia a los contextos y los entornos para las personas y el impacto que estos generan para la salud. En este sentido, y de acuerdo con la propuesta del modelo salutogénico, los recursos generales de resistencia se basan en actividades significativas para las personas que les aportan valor y significado y que se realizan en su entorno cercano (Hernán et al., 2013; Mittelmark et al., 2017). En esta línea, los planteamientos de la disciplina de terapia ocupacional son afines a las propuestas de los activos para la salud. De este modo, es importante considerar los beneficios de los activos para la salud para el desempeño ocupacional desarrollando una visión sobre las ocupaciones de la población joven teniendo en cuenta que este colectivo se encuentra en una etapa vital determinante. Así, la adolescencia, es la etapa vital en la que se establecen los hábitos de vida y se inician algunas conductas de riesgo para la salud (Hernán et al., 2013) y por este motivo es fundamental identificar los activos para la salud de este grupo poblacional.

Teniendo esto en cuenta, el principal objetivo de este artículo es revisar la literatura sobre los principales activos para la salud en población joven desde una perspectiva ocupacional con la finalidad de identificar cuáles son las ocupaciones o actividades más significativas.

\section{Metodología}

\section{Diseño}

Se lleva a cabo una búsqueda exploratoria usando Google-Académico con la finalidad de definir el tema y el enfoque de esta revisión bibliográfica. Posteriormente, se realiza una segunda búsqueda para responder a los objetivos planteados en esta revisión en la que se exploran las siguientes bases de datos: Pubmed, Biblioteca Virtual en Salud y ScienceDirect 
(Elsevier). Por otro lado, se ha identificado Dialnet, ÍnDICEs CSIC (Consejo Superior Investigaciones Científicas), SciELO (Scientific Electronic Library Online) y JSTOR (abreviatura en inglés de Journal STORage) como bases de datos que pueden ser de interés, pero no se ha podido encontrar literatura sobre activos para la salud relacionada con la población de interés en estas.

\section{Estrategia de búsqueda}

En las bases de datos de las cuales se ha extraído información relevante para la revisión: Pubmed, Biblioteca Virtual en Salud y ScienceDirect (Elsevier), se han realizado las búsquedas descritas en la Tabla 1. Además, se ha hecho uso de los descriptores boleanos AND u OR para combinar las palabras identificadas para cada concepto clave, con el fin de precisar la búsqueda o ampliar el número de resultados encontrados. También se ha usado entrecomillado para los conceptos que se componen por más de una palabra; como por ejemplo "activos para la salud", "prescripción social" o "terapia ocupacional". Otros tipos de filtros de búsqueda utilizados fueron la fecha de publicación (artículos de los últimos 5 años) y la búsqueda por palabras clave en título y abstract.

Tabla 1. Resultados obtenidos en las bases de datos consultadas.

\begin{tabular}{cccc}
\hline Base de datos & Ecuación de búsqueda & $\begin{array}{c}\text { No artículos } \\
\text { encontrados }\end{array}$ & $\begin{array}{c}\text { No artículos } \\
\text { seleccionados }\end{array}$ \\
\hline PubMed & $\begin{array}{c}\text { ("health assets"[Title/Abstract]) AND } \\
\text { (Children OR Adolescents } \\
\text { [Title/Abstract] })\end{array}$ & 16 & 2 \\
\hline $\begin{array}{c}\text { ScienceDirect } \\
\text { (Elsevier) }\end{array}$ & $\begin{array}{c}\text { "health assets" AND (Children OR } \\
\text { Adolescents) }\end{array}$ & 123 & 2 \\
\hline $\begin{array}{c}\text { Biblioteca virtual en } \\
\text { salud }\end{array}$ & "Activos para la salud" AND jóvenes & 162 & 2 \\
\hline
\end{tabular}

El proceso de selección de los artículos consistió en un cribado inicial en la búsqueda en función del año de publicación ( $<5$ años), que el tema esté presente en el título o abstract, que hablen de la ocupación como un aspecto de prevención/promoción de la salud y que se centre en el colectivo de jóvenes; como criterios de inclusión. Una vez hecha la primera selección de resultados, se realizó una primera lectura de estos en los que se eliminaron aquellos que no estaban relacionados con el foco de estudio.

Con relación a las dificultades de acceso se han identificado dos tipos. Por un lado, no se ha podido acceder a algunas bases de datos ya que se necesita subscripción a estas para poder realizar una búsqueda. Por otro lado, algunas bases de datos consultadas solo permitían realizar una búsqueda simple, por lo que no ayudaban a concretar la búsqueda y encontrar los documentos de interés para la revisión (Figura 1). 


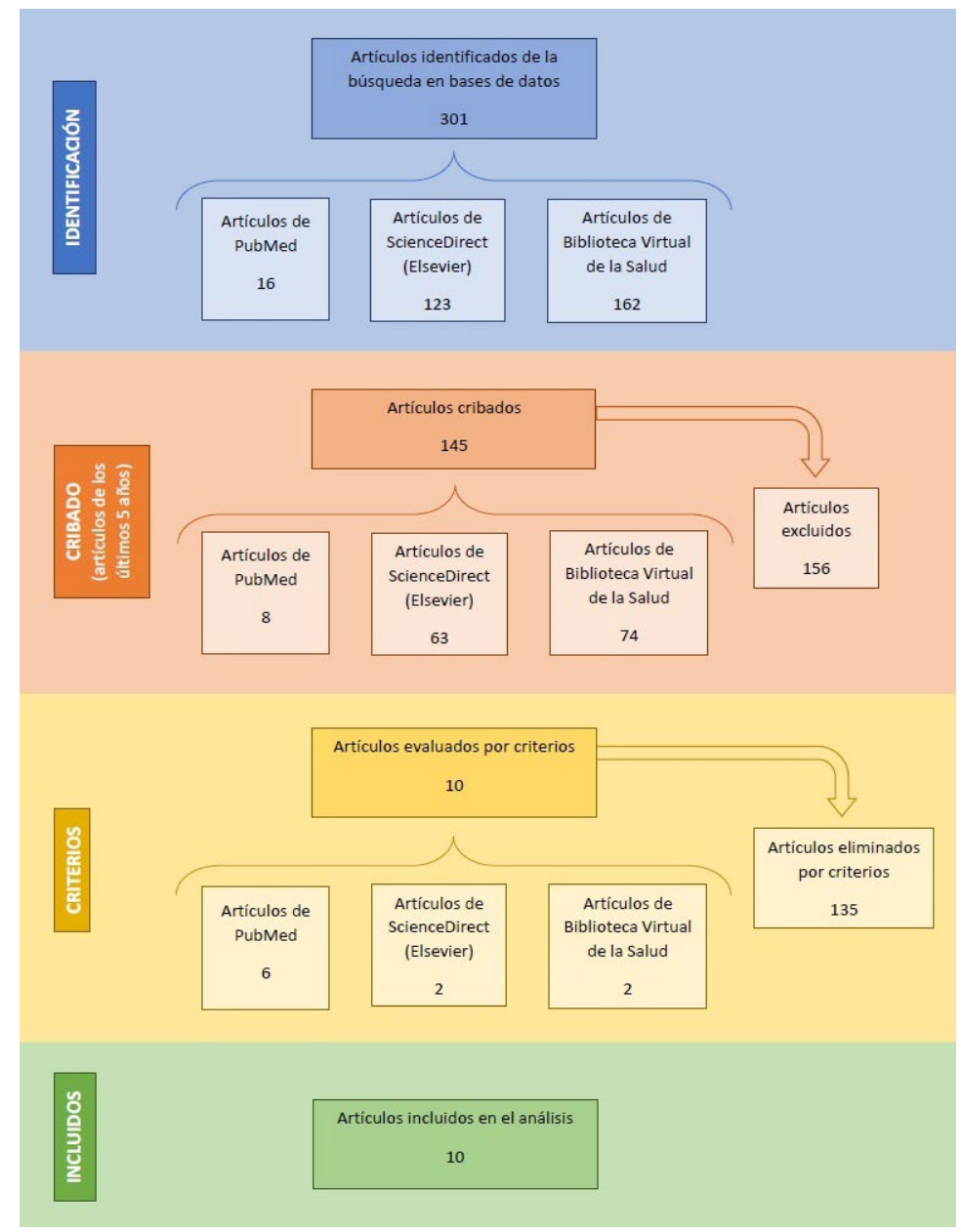

Figura 1. Artículos escogidos para la revisión a partir de la búsqueda bibliográfica. Elaboración propia.

\section{Extracción de datos}

A partir de los objetivos establecidos, se construyó una matriz de recogida de datos (ver Tabla 2) para identificar y redactar los resultados obtenidos de la literatura y elaborar una discusión mediante el análisis de los resultados identificados sobre los activos para la salud y el impacto que tienen estos en el desempeño ocupacional de los jóvenes.

Esta revisión de la literatura, pretende enriquecer los estudios ya realizados sobre activos para la salud aportando la visión de la terapia ocupacional; esto permitiría enfatizar la ocupación en la teoría de los activos para la salud. Por otra parte, se pretende identificar el valor añadido que pueden aportar las bases de la terapia ocupacional comunitaria a la teoría de los activos para la salud, con el objetivo de potenciar los beneficios en el desempeńo ocupacional de los jóvenes al aplicar estas intervenciones contando con un terapeuta ocupacional en el equipo.

\section{Resultados}

Finalmente, los artículos seleccionados se recogen en la tabla de selección (Tabla 2). 
Tabla 2. Tabla artículos seleccionados.

\begin{tabular}{|c|c|c|c|c|}
\hline AUTOR Y AÑO & $\begin{array}{l}\text { BASE DE } \\
\text { DATOS }\end{array}$ & PAÍS & METODOLOGÍA & TEMA PRINCIPAL \\
\hline $\begin{array}{l}\text { Calmeiro et al. } \\
\text { (2018) }\end{array}$ & PubMed & Portugal & $\begin{array}{c}\text { Se recogen encuestas de } 3494 \\
\text { participantes de } 8^{\circ} \text { y } 10^{\circ} \text { grado de } 139 \\
\text { diferentes escuelas de Portugal. }\end{array}$ & $\begin{array}{c}\text { Satisfacción de vida percibida por } \\
\text { los adolescentes y activos para la } \\
\text { salud. }\end{array}$ \\
\hline $\begin{array}{l}\text { Moreno } \\
\text { Sánchez et al. } \\
\text { (2017) }\end{array}$ & $\begin{array}{l}\text { ScienceDirect } \\
\text { (Elsevier) }\end{array}$ & España & $\begin{array}{c}\text { La propuesta es generar un mapa de } \\
\text { activos sostenidos por el potencial y los } \\
\text { intereses de las comunidades. La } \\
\text { muestra es de } 106 \text { personas de } 5 \text { a } 18 \\
\text { ańos. }\end{array}$ & $\begin{array}{l}\text { Plantear intervención para } \\
\text { aumentar la conciencia de los } \\
\text { estilos de vida. }\end{array}$ \\
\hline $\begin{array}{c}\text { Sáenz Mendía et al. } \\
\text { (2014) }\end{array}$ & $\begin{array}{l}\text { ScienceDirect } \\
\text { (Elsevier) }\end{array}$ & España & $\begin{array}{c}\text { Diseño cualitativo en una } \\
\text { investigación de acción participativa } \\
\text { con } 30 \text { participantes, siendo estos } \\
\text { profesores, padres y profesionales de la } \\
\text { salud que están en contacto con niños } \\
\text { de } 8 \text { a } 9 \text { años }\end{array}$ & $\begin{array}{l}\text { Modelo de activos para la salud } \\
\text { en una comunidad de } \\
\text { aprendizaje. }\end{array}$ \\
\hline $\begin{array}{l}\text { Vera- } \\
\text { Remartínez et al. } \\
(2017)\end{array}$ & $\begin{array}{c}\text { Biblioteca virtual } \\
\text { en salud }\end{array}$ & España & $\begin{array}{c}\text { Se realizó un análisis descriptivo } \\
\text { transversal, con una etapa cuantitativa } \\
\text { y otra cualitativa, en la que se pasaron } \\
\text { varias pruebas y participaron } 124 \\
\text { jóvenes varones de } 18 \text { a } 22 \text { años, de los } \\
\text { tres centros penitenciarios de la región. }\end{array}$ & $\begin{array}{l}\text { Determinar el sentido de } \\
\text { coherencia (SOC) en jóvenes } \\
\text { presos y elaborar un mapa de } \\
\text { activos para la salud. }\end{array}$ \\
\hline $\begin{array}{l}\text { Hernán García et al. } \\
\text { (2015b) }\end{array}$ & $\begin{array}{c}\text { Biblioteca virtual } \\
\text { en salud }\end{array}$ & España & $\begin{array}{l}\text { Usando técnicas de etnografía virtual: } \\
\text { consultan a } 55 \text { estudiantes de } \\
\text { enseńanza secundaria en foros virtuales } \\
\text { y a } 21 \text { estudiantes universitarios en } \\
\text { video entrevistas semi-estructuradas. }\end{array}$ & $\begin{array}{l}\text { Conocer la opinión de los } \\
\text { estudiantes sobre la relación entre } \\
\text { el internet y la salud. }\end{array}$ \\
\hline $\begin{array}{l}\text { Hernán-García et al. } \\
\text { (2015a) }\end{array}$ & PubMed & España & $\begin{array}{l}\text { Estudio cualitativo basado en } 8 \text { grupos } \\
\text { focales que comprenden } 64 \text { alumnos } \\
\text { de } 8 \text { escuelas primarias en la región de } \\
\text { Andalucía, Espańa. }\end{array}$ & $\begin{array}{l}\text { Opiniones de alumnos de } \\
\text { primaria sobre el internet como } \\
\text { fuente de activos para la salud y el } \\
\text { bienestar. }\end{array}$ \\
\hline Klemera et al. (2017) & PubMed & Inglaterra & $\begin{array}{c}1608 \text { encuestados de } 15 \text { años como } \\
\text { parte del Estudio de Comportamiento } \\
\text { de Salud de la OMS en Niños de } \\
\text { Inglaterra. }\end{array}$ & $\begin{array}{l}\text { Examinar si los entornos del } \\
\text { adolescente podrían funcionar } \\
\text { como activos para la salud } \\
\text { protectores contra el } \\
\text { comportamiento autolesivo. }\end{array}$ \\
\hline $\begin{array}{l}\text { Soriano-Ayala et al. } \\
\qquad(2020)\end{array}$ & PubMed & España & $\begin{array}{l}\text { El estudio utiliza el “photovoice”, un } \\
\text { método en que los jóvenes realizan } \\
\text { fotografías a aquello que creen que son } \\
\text { activos para su salud, posteriormente } \\
\text { se hacen entrevistas individuales en las } \\
\text { que se realizan preguntas sobre las } 6 \\
\text { fotografías seleccionadas como más } \\
\text { relevantes. El estudio utiliza una } \\
\text { metodología cualitativa. }\end{array}$ & $\begin{array}{c}\text { Identificar activos transculturales } \\
\text { y culturales de jóvenes rumanos, } \\
\text { marroquíes y españoles. }\end{array}$ \\
\hline $\begin{array}{c}\text { Pérez-Wilson et al. } \\
\text { (2015) }\end{array}$ & PubMed & España & $\begin{array}{l}20 \text { entrevistas semiestructuradas, } 2 \\
\text { grupos focales con adolescentes y } 4 \\
\text { entrevistas semiestructuradas con } \\
\text { informantes clave ( } 4 \text { adultos que } \\
\text { trabajan con adolescentes). }\end{array}$ & $\begin{array}{l}\text { Perspectivas de los jóvenes sobre } \\
\text { la salud, el bienestar y sus } \\
\text { determinantes. }\end{array}$ \\
\hline $\begin{array}{c}\text { Paakkari et al. } \\
\text { (2019) }\end{array}$ & PubMed & Finlandia & $\begin{array}{l}\text { Se obtuvo una muestra representativa } \\
\text { de las escuelas de habla finlandesa, } \\
\text { compuesta por } 3833 \text { adolescentes de } \\
13 \text { y } 15 \text { años de } 359 \text { escuelas } \\
\text { diferentes. Se aplicó el análisis de } \\
\text { conglomerados en dos pasos y la } \\
\text { regresión logística binaria multinivel } \\
\text { de efectos mixtos. }\end{array}$ & $\begin{array}{l}\text { Asociaciones entre los activos de } \\
\text { salud de los adolescentes y varios } \\
\text { indicadores de salud (tabaquismo, } \\
\text { consumo de alcohol, duración del } \\
\text { sueńo, actividad física, } \\
\text { alimentación saludable, salud } \\
\text { oral, salud autoinformada, } \\
\text { múltiples quejas de salud). }\end{array}$ \\
\hline
\end{tabular}

Tras la revisión de los artículos que cumplían con los criterios de inclusión se han considerado aquellas ocupaciones o actividades significativas más relevantes para los 
jóvenes. Además, la mayoría de los activos para la salud identificados han sido reconocidos por los jóvenes con lo que ello implica para los abordajes de terapia ocupacional. A continuación, se presenta por apartados los aspectos más destacables de la revisión.

\section{Población destinataria de los abordajes planteados}

En la revisión se exploran dos estudios (Hernán García et al., 2015b; VeraRemartínez et al., 2017) en los que la población ha cumplido la mayoría de edad, llegando hasta los 22 años. Hernán García et al. (2015b), además, añade a estudiantes de educación secundaria en su estudio. Según la bibliografía explorada, la mayoría de los estudios cotejados se centran en la franja de edad de los estudiantes de secundaria (de 12 a 18 ańos).

Por otro lado, el estudio de Moreno Sánchez et al. (2017), añade a los niños de primaria como foco de su estudio. A diferencia de Hernán-García et al. (2015a) y Sáenz Mendía et al. (2014), que exploran los activos para la salud únicamente en menores de 5 a 12 ańos; el primero realiza el estudio a partir de grupos focales formados por los propios alumnos, mientras que Sáenz Mendía et al. (2014) se centra en la comunidad educativa que está en contacto con los niños: padres, profesores y profesionales de la salud, realizando una investigación de acción participativa. Pérez-Wilson et al. (2015) también tienen en cuenta los adultos que están en contacto con los adolescentes, a los que realizan entrevistas semiestructuradas; mientras que, con los adolescentes, a parte de las entrevistas se realizan dos grupos focales.

\section{Tipos de metodologías empleadas}

En cuanto a la metodología, los estudios explorados han aplicado diferentes métodos: encuestas (Calmeiro et al., 2018; Klemera et al., 2017), mapa de activos (Moreno Sánchez et al., 2017), "photovoice" (Soriano-Ayala et al., 2020) investigación de acción participativa (Sáenz Mendía et al., 2014), foros virtuales y video entrevistas semiestructuradas (Hernán García et al., 2015b), grupos focales (Hernán-García et al., 2015a; PérezWilson et al., 2015), entrevistas semiestructuradas (Soriano-Ayala et al., 2020; PérezWilson et al., 2015) y diferentes pruebas (Vera-Remartínez et al., 2017; Paakkari et al., 2019).

\section{Activos para la salud en jóvenes}

En cuanto a los activos para la salud identificados en la literatura explorada, se han observado diferentes clasificaciones; en este caso, se ofrece una clasificación centrada en la persona, la ocupación y el entorno (Figura 2), con la finalidad de poner de forma gráfica referida los activos para la salud más significativos en los jóvenes desde una perspectiva ocupacional.

En relación con la persona, encontramos que los valores, la fuerza de voluntad, la autoestima, la autorregulación, los recursos personales, el bienestar psicosocial, el autoconocimiento, el sentido del humor, la resistencia a las presiones, la autoconfianza, el estado de ánimo positivo, la autoeficacia y la capacidad de manejar dificultades y desafíos se perciben como activos para la salud (Calmeiro et al., 2018; Hernán García et al., 2015b; Moreno Sánchez et al., 2017; Pérez-Wilson et al., 2015; Sáenz Mendía et al., 2014; Soriano-Ayala et al., 2020; Vera-Remartínez et al., 2017). También se considera un activo la competencia social, el respeto, la cooperación, la capacidad de expresión y la capacidad de hablar con otras personas sobre sus sentimientos (Calmeiro et al., 2018; Hernán García et al., 2015b; Pérez-Wilson et al., 2015; Soriano-Ayala et al., 2020; VeraRemartínez et al., 2017). 


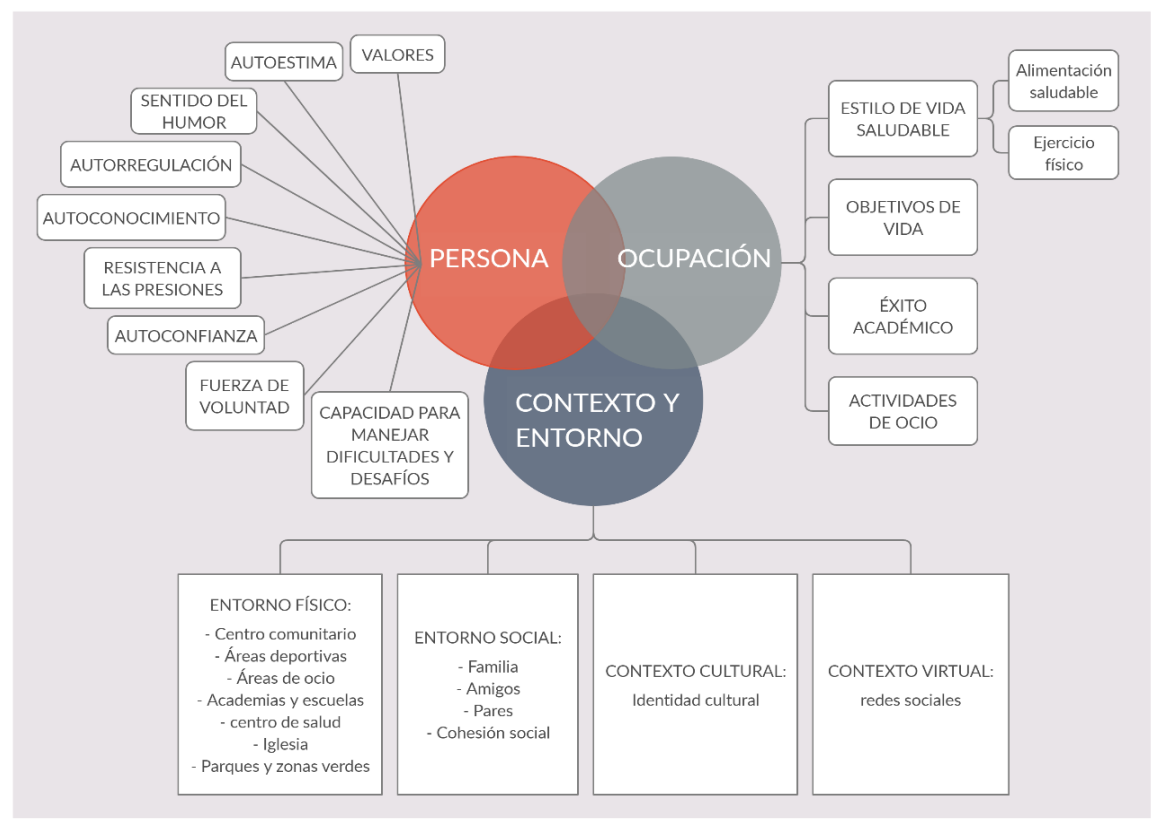

Figura 2. Activos para la salud identificados por jóvenes. Elaboración propia.

Los activos identificados relacionados con la ocupación son (según Calmeiro et al., 2018; Hernán-García et al., 2015a; Pérez-Wilson et al., 2015; Sáenz Mendía et al., 2014; Soriano-Ayala et al., 2020; Vera-Remartínez et al., 2017), en primer lugar: llevar un estilo de vida saludable (alimentación saludable y ejercicio físico); en segundo lugar, encontramos los objetivos de vida y el éxito académico; y, por último, el ocio (internet, juego, lectura, etc.). Por otro lado, Sáenz Mendía et al. (2014) y Soriano-Ayala et al. (2020) en sus artículos, nos destacan la autonomía como un activo para la salud.

En relación con la persona, encontramos que los valores, la fuerza de voluntad, la autoestima, la autorregulación, los recursos personales, el bienestar psicosocial, el autoconocimiento, el sentido del humor, la resistencia a las presiones, la autoconfianza, el estado de ánimo positivo, la autoeficacia y la capacidad de manejar dificultades y desafíos se perciben como activos para la salud (Calmeiro et al., 2018; Hernán García et al., 2015b; Moreno Sánchez et al., 2017; Pérez-Wilson et al., 2015; Sáenz Mendía et al., 2014; Soriano-Ayala et al., 2020; Vera-Remartínez et al., 2017). También se considera un activo la competencia social, el respeto, la cooperación, la capacidad de expresión y la capacidad de hablar con otras personas sobre sus sentimientos (Calmeiro et al., 2018; Hernán García et al., 2015b; Pérez-Wilson et al., 2015; Soriano-Ayala et al., 2020; VeraRemartínez et al., 2017).

Los activos identificados relacionados con la ocupación son (según Calmeiro et al., 2018; Hernán-García et al., 2015a; Pérez-Wilson et al., 2015; Sáenz Mendía et al., 2014; Soriano-Ayala et al., 2020; Vera-Remartínez et al., 2017), en primer lugar: llevar un estilo de vida saludable (alimentación saludable y ejercicio físico); en segundo lugar, encontramos los objetivos de vida y el éxito académico; y, por último, el ocio (internet, juego, lectura, etc.). Por otro lado, Sáenz Mendía et al. (2014) y Soriano-Ayala et al. (2020) en sus artículos, nos destacan la autonomía como un activo para la salud. 
Para identificar los activos para la salud que se encuentran en el entorno y contexto de los adolescentes, se sigue la definición de estos establecida por el Marco de trabajo de la práctica de terapia ocupacional de la AOTA (Álvarez et al., 2010):

- Entorno físico: se consideran activos para la salud: el centro comunitario, las áreas deportivas, las áreas de ocio, las academias y escuelas, el centro de salud, la iglesia y los parques y áreas verdes (Morgan \& Ziglio, 2007 en Moreno Sánchez et al., 2017; PérezWilson et al., 2015; Soriano-Ayala et al., 2020; Vera-Remartínez et al., 2017).

- Entorno social: las relaciones positivas ofrecen estabilidad emocional y afecto a la persona, es el caso de la familia, los amigos y los pares (Calmeiro et al., 2018; Klemera et al., 2017; Morgan \& Ziglio, 2007 en Moreno Sánchez et al., 2017; Soriano-Ayala et al., 2020; Vera-Remartínez et al., 2017). La familia ofrece apoyo y es un ejemplo para las conductas presentes y futuras; por otro lado, los amigos y los pares conforman la red social de los adolescentes y aumentan la autoestima, la motivación y el disfrute (Hernán García et al., 2015b; Pérez-Wilson et al., 2015; Sáenz Mendía et al., 2014). La socialización, conectividad escolar y la cohesión comunitaria, tanto de la comunidad como del vecindario, también son vistos como activos para la salud por los adolescentes (Calmeiro et al., 2018; Klemera et al., 2017; SorianoAyala et al., 2020).

- Contexto cultural: la tolerancia cultural se valora como un activo para la salud (Morgan \& Ziglio, 2007 en Moreno Sánchez et al., 2017). Culturalmente los adolescentes españoles ven los medicamentos como activos para la salud, mientras que los adolescentes marroquíes le dan más valor a la medicina tradicional y los adolescentes rumanos a los remedios caseros (Soriano-Ayala et al., 2020). Además, los adolescentes marroquíes valoran la identidad cultural, como la forma de vestir o la comida de la cultura de origen, como activos para la salud (Soriano-Ayala et al., 2020). Por otro lado, Soriano-Ayala et al. (2020) identifican que las mujeres rumanas y españolas, por influencia de la sociedad, consideran la delgadez como un activo para la salud.

- Contexto virtual: los adolescentes consideran que las redes sociales son un activo para la salud (Soriano-Ayala et al., 2020).

En cuanto a la salud, los adolescentes, según los estudios explorados, la definen como un concepto integral, que engloba dimensiones físicas, psicológicas y sociales, que se asocia a la felicidad y la calidad de vida (Hernán García et al., 2015b; Pérez-Wilson et al., 2015).

Los activos para la salud que presentan una mayor significatividad para el bienestar de los adolescentes tienen que ver con la socialización y la relación con los otros (capital social), ya que ofrece una variedad de recursos para el desarrollo personal (Calmeiro et al., 2018; Klemera et al., 2017). En una sociedad donde el vecindario está asociado al patrimonio y a la cultura, es importante sentirse valorado por la comunidad (Murphey et al., 2004 en Calmeiro et al., 2018; Pérez-Wilson et al., 2015).

En estos artículos se ha identificado un fuerte impacto de la relación con la familia para los activos para la salud (Calmeiro et al., 2018; Klemera et al., 2017; Soriano-Ayala et al., 2020). En el caso de Soriano-Ayala et al. (2020), han observado que el significado de este activo varía según la cultura; para las personas migrantes la familia genera un rol de cohesión social, mientras que en el grupo español la familia es vista como un soporte emocional y económico. 


\section{Diferencia con las personas migrantes}

En el estudio que se realizó con personas internas en la comunidad de Valencia (VeraRemartínez et al., 2017), se observa que los internos migrantes presentaban un SOC mayor a los espańoles, lo cual plantea la hipótesis de que las experiencias exitosas previas y actuales para afrontar situaciones adversas influyen en el SOC y aumentan la capacidad y la actitud proactiva para aprovechar los recursos que ofrece la comunidad. Por otra parte, las personas que vivían en centros penitenciarios que contaban con una mayor oferta de actividades escolares y deportivas, presentaban un mayor SOC que los otros internos (VeraRemartínez et al., 2017). Sáenz Mendía et al. (2014) analizan el modelo de activos para la salud en una comunidad de aprendizaje en Pamplona (España) e identifican que la diversidad, la educación inclusiva y la cooperación son valores positivos en la escuela.

Para finalizar los resultados de esta revisión, cabe destacar que Soriano-Ayala et al. (2020) consideran que algunos activos para la salud pueden surgir derivados de influencias de la sociedad y que estos pueden no desencadenar el goce de una buena salud para el adolescente (como las drogas o la delgadez); así pues, consideran importante iniciar la acción educativa cuestionando los activos. En Paakkari et al. (2019) determinan que las intervenciones de promoción y protección de la salud deben desarrollar varios activos para la salud, con el fin de que los adolescentes cuenten con múltiples activos para evitar desarrollar comportamientos de riesgo. Es importante la involucración y la participación de la comunidad en la búsqueda de activos para la salud para que todos logren el mayor nivel de bienestar (Sáenz Mendía et al., 2014).

\section{Discusión}

Pese a encontrar escasa literatura sobre terapia ocupacional en activos para la salud en los jóvenes, actualmente terapeutas ocupacionales del estado están realizando estudios sobre los activos para la salud. Es el caso de Ma Isabel Vidal Sánchez que, juntamente con tres compañeros más, encabezó una experiencia piloto sobre los activos para la salud mental en dos centros educativos de Torrero (Vidal et al., 2017). En esta experiencia también se destaca la autonomía y las relaciones sociales, valoradas igualmente por los autores revisados en este trabajo. Y se remarca, en ambos casos, que los menores de hoy en día tienen una visión más holística de la salud. Por último, Ma Isabel Vidal Sánchez también secunda la visión, ofrecida por Soriano-Ayala et al. (2020), de que los activos para la salud tienen influencias de los valores sociales imperantes en la actualidad.

Por otra parte, el tema del internet como activo para la salud tiene algunas controversias. Por esa razón, en los artículos de Hernán García et al. (2015a, 2015b) quieren destacar la importancia de la alfabetización digital y sanitaria, para navegar por internet de manera positiva aumentando la calidad y credibilidad del contenido. Por otra parte, el artículo "Identificación de activos culturales y transculturales de salud entre adolescentes marroquíes, rumanos y españoles a través del método Photovoice" se da importancia a las redes sociales para poder seguir en contacto con sus familiares y amigos (SorianoAyala et al., 2020).

Además, el Colegio de Terapeutas Ocupacionales de Catalunya (COTOC) recientemente ha publicado un documento en el que habla de los programas de prescripción social, como medida para disminuir el riesgo de aislamiento social y alternativa a la medicalización. Relata, a partir de la Agència de Salut Pública de Catalunya (2019 en 
Busquets et al. (2020), los beneficios de estos programas basados en la participación en actividades de su entorno local. En este mismo documento, se compara estos programas de prescripción social con la prescripción ocupacional, ya que ambos usan las actividades y los recursos como medio para mejorar la salud de las personas (Busquets et al., 2020).

La prescripción ocupacional parte del concepto de salud ocupacional, que viene determinada por el equilibrio entre el desempeño de la persona, su proyecto y estilo de vida y las demandas y exigencias del entorno (Busquets et al., 2020). Para llevar a cabo la prescripción ocupacional se establece un plan de acción para cubrir las necesidades de la persona. Para realizarlo, se evalúan dimensiones ocupacionales, del entorno y del contexto, y se realiza el perfil ocupacional de la persona (Busquets et al., 2020).

Por tanto, el terapeuta ocupacional realiza un análisis del activo para la salud, desde las demandas y características de la propia actividad hasta el estado de salud de la persona, su entorno físico y social, su pasado y sus propias proyecciones de futuro (Busquets et al., 2020). Es importante graduar y adaptar las actividades a la situación personal y colectiva en cada realidad individual, para evitar que las personas dejen la actividad o no lleguen a realizarla. Así pues, se pretende prevenir que se reproduzcan los resultados de las pruebas piloto de prescripción social en Atención Primaria en Cataluña, donde se afirma que el $49,22 \%$ de las personas no se vincularon a ninguna actividad (Capella, 2019 en Busquets et al., 2020). Además, según un artículo publicado en el Royal College of Occupational Therapists (2015 en Busquets et al., 2020), la terapia ocupacional aporta numerosos beneficios a la atención primaria, como la prevención de recaídas, la disminución de los ingresos hospitalarios y el retraso de la institucionalización a largo plazo.

Como limitaciones de esta revisión, se ha podido tener interferencias por el idioma de alguno de los artículos, el cual no hablo con fluidez y puede haber afectado a la comprensión de estos. La búsqueda la he valorado como una limitación, ya que era muy concreta y puede que haya descartado artículos importantes a la hora de aplicar criterios de inclusión y exclusión. Por el contrario, también se valora como una fortaleza ya que me ha ayudado a concretar el tema a tratar. Finalmente, el haber encontrado activos que identificaran los propios jóvenes en estudios diversos creo que es una fortaleza y que ha enriquecido el trabajo en cuanto a los activos más valorados por ellos, pienso que es una recopilación que puede resultar útil para futuras intervenciones en jóvenes.

\section{Conclusiones}

Después de realizar la revisión, se constata que los activos para la salud más relevantes en los jóvenes han sido el autoconocimiento y la autoconfianza. En cuanto a la ocupación, consideran que llevar un estilo de vida saludable, tener objetivos de vida y realizar actividades de ocio son activos para la salud. También han considerado como activos la socialización y la relación con los otros, dando especial valor a la familia como ejemplo de conductas y apoyo emocional. Además de los espacios físicos, toma relevancia la cultura de procedencia y las redes sociales.

Por otra parte, se ha observado que los activos para la salud descritos por los jóvenes pueden presentar influencias por parte de la sociedad que, en vez de aportar salud, pueden ser nocivas para ellos. Para ello, una vez identificados estos activos, se propone cuestionarlos y potenciar una visión crítica para poder discernir en los que realmente aportan salud y bienestar y los que, por el contrario, no. 
A partir de este trabajo se abren posibles investigaciones. Por un lado, determinar si la identificación de los activos para la salud por parte de la comunidad conduce a reducir las desigualdades entre las diferentes personas. Por otro lado, resolver si el porcentaje de vinculación a los activos para la salud aumenta en los equipos que cuenten con un terapeuta ocupacional que realice un análisis del activo, el entorno y la persona y, así, aumentar la vinculación de las personas a los activos presentados por los profesionales de los Centros de Atención Primaria.

\section{Referencias}

Algado, S. S. (2015). Una terapia ocupacional desde un paradigma crítico. TOG (A Coruña), 7, 25-40.

Álvarez, A. A., Canosa, N., Groba, B., Martínez, R., Matilla, R., Bocanegra, N. M., Méndez, B. M., Rivas, N., Talavera, M., \& Viana, I. (2010). Marco de trabajo para la práctica de la terapia ocupacional: dominio y proceso. Puerto Rico: AOTA.

Busquets, M., Domingo, E., \& Guzmán, S. (2020). Consultoría ocupacional y prescripción ocupacional. Barcelona: Colegio de Terapeutas Ocupacionales de Catalunya.

Calmeiro, L., Camacho, I., \& Matos, M. G. (2018). Life satisfaction in adolescents: the role of individual and social health assets. The Spanish Journal of Psychology, 21(23), 1-8. http://dx.doi.org/10.1017/sjp.2018.24.

Cantero Garlito, P. A. (2018). Terapia ocupacional comunitaria: construyendo en, con y desde la comunidad. Recuperado el 24 de febrero de 2021, de https://ocupandolosmargenes.org/terapia-ocupacionalcomunitaria-construyendo-desde-la-comunidad/

Cofiño, R., Aviñó, D., Benedé, C. B., Botello, B., Cubillo, J., Morgan, A., Paredes-Carbonell, J. J., \& Hernán, M. (2016). Promoción de la salud basada en activos: ¿cómo trabajar con esta perspectiva en intervenciones locales?. Gaceta Sanitaria, 30(1), 93-98. http://dx.doi.org/10.1016/j.gaceta.2016.06.004.

Hernán, M., Morgan, A., \& Mena, Á. L. (2013). Formación en salutogénesis y activos para la salud. Andalucía: Escuela Andaluza de Salud Pública, Consejería de Salud y Bienestar Social.

Hernán-García, M., Botello-Díaz, B., Marcos-Marcos, J., Toro-Cárdenas, S., \& Gil-García, E. (2015a). Understanding children: a qualitative study on health assets of the Internet in Spain. International Journal of Public Health, 60(2), 239-247. http://dx.doi.org/10.1007/s00038-015-0648-0.

Hernán García, M., Toro Cárdenas, S., Leralta Piñan, O., Pérez Trigueros, M., Carrasco Campos, R., \& Lineros González, C. (2015b). El Internet como fuente de información sobre la salud: la visión de estudiantes de Andalucía, España. Global Health Promotion, 22(1), 100-109.

Klemera, E., Brooks, F. M., Chester, K. L., Magnusson, J., \& Spencer, N. (2017). Self-harm in adolescence: protective health assets in the family, school and community. International Journal of Public Health, 62(6), 631-638. http://dx.doi.org/10.1007/s00038-016-0900-2.

Mittelmark, M. B., Sagy, S., Eriksson, M., Bauer, G. F., Pelikan, J. M., Lindström, B., \& Espnes, G. A. (2017). The Handbook of Salutogenesis. Suiza: Springer.

Moreno Sánchez, E., Castillo Viera, E., \& Rodríguez-Mena, J. A. (2017). A cross-border cooperation project between spain and portugal to improve the quality of life of the population in primary school. Procedia: Social and Behavioral Sciences, 237, 438-445. http://dx.doi.org/10.1016/j.sbspro.2017.02.087.

Observatorio de Salud de Asturias. (2017). Guía ampliada para la recomendación de activos ("prescripción social”) en el sistema sanitario. Asturias: Principado de Asturias.

Paakkari, L., Torppa, M., Välimaa, R., Villberg, J., Ojala, K., \& Tynjälä, J. (2019). Health asset profiles and health indicators among 13- and 15-year-old adolescents. International Journal of Public Health, 64(9), 1301-1311. http://dx.doi.org/10.1007/s00038-019-01280-7.

Pérez-Wilson, P., Hernán, M., Morgan, A. R., \& Mena, A. (2015). Health assets for adolescents: opinions from a neighbourhood in Spain. Health Promotion International, 30(3), 552-562. http://dx.doi.org/10.1093/heapro/dat081. 
Royal College of Occupational Therapists - RCOT. (2017). Professional standards for occupational therapy practice. Londres: College of Occupational Therapists.

Sáenz Mendía, R., Gabari Gambarte, M. I., Idoate García, V., \& García García, J. M. (2014). Assets in a community school health. Procedia: Social and Behavioral Sciences, 132(15), 615-620. http://dx.doi.org/10.1016/j.sbspro.2014.04.362.

Sociedad Española de Salud Pública y Administración Sanitaria - SESPAS. (2017). En esta tierra hay algo qye merece vivir. In Anales de Congreso de Promoción de la Salud: Activos para la Salud Comunitaria. Granada: Escuela Andaluza de Salud Pública de Granada.

Soriano-Ayala, E., Cala, V. C., \& Ruiz-Salvador, D. (2020). Identification of cultural and transcultural health assets among moroccan, romanian and spanish adolescents through photovoice. Journal of Immigrant and Minority Health, 22(2), 255-265. http://dx.doi.org/10.1007/s10903-019-00934-1.

Souto Gómez, A. I., Talavera Valverde, M. A., Facal Fondo, T., \& Rodríguez Otero, L. M. (2019). Terapia ocupacional y promoción de la salud. Madrid: Editorial Síntesis.

Vera-Remartínez, E. J., Paredes-Carbonell, J. J., Aviñó Juan-Ulpiano, D., Jiménez-Pérez, M., Araujo Pérez, R., Agulló-Cantos, J. M., \& Mora Notario, A. (2017). Sentido de coherencia y mapa de activos para la salud en jóvenes presos de la Comunidad Valenciana en Espańa. Global Health Promotion, 24(3), 112-121.

Vidal, M. I., Gasch, A., Araguás, A., \& Tomás, C. (2017). Activos para la salud mental de la infancia de Torrero: experiencia piloto en dos centros educativos. In Anales del Congreso Activos para la Salud Comunitaria (pp. 466-468). Granada: Escuela Andaluza de Salud Pública.

Zango Martín, I. (2011). Definición de terapia ocupacional: lo que soy y lo que hago. TOG (A Coruña), 4, 62-68.

Zango Martín, I. (2017). Terapia Ocupacional Comunitaria. Madrid: Editorial Síntesis.

\section{Contribución de los Autores}

Rut Alsina-Santana ha realizado el texto y organizado las fuentes y análisis. Inmaculada Zango-Martín ha dado soporte durante todo el proceso realizando la revisión del texto, así como la organización del análisis planteado. Todos los autores aprueban la versión final del texto.

\section{Autor para la correspondencia}

Rut Alsina-Santana

e-mail: alsina.rut@gmail.com

\section{Editor de sección}

Prof. Dr. Vagner dos Santos 